\title{
CALCULATION OF CAPILLARY CONDUCTIVITY FROM PRESSURE PLATE OUTFLOW DATA WITH NON- NEGLIGIBLE MEMBRANE IMPEDANCE ${ }^{1}$ )
}

\author{
P. E. RIJTEMA \\ Institute for Land and Water Management Research, \\ Wageningen, The Netherlands
}

\begin{abstract}
SUMMary
The flow equation describing the movement of water out of a soil sample placed in a pressure plate apparatus, is solved for the case that the impedance of the membrane or porous plate will have to be taken into account. A practical and simple method is developed for the calculation of the capillary conductivity from outflow data. Using this method the total impedance at the lower boundary of the sample is determined from the experimental outflow data for each pressure step applied. In this way it is not necessary to determine the membrane impedance experimentally. The influence of the membrane impedance on the final value of the capillary conductivity is computed for various values of the impedance.
\end{abstract}

\section{INTRODUCTION}

Measurements of soil moisture conditions in the field have shown that water is distributed throughout the root zone of plants largely by means of unsaturated flow. The measure of influence of the unsaturated flow on evapotranspiration during periods, when the soil moisture is being depleted, is still partly a matter of speculation. A study of the unsaturated flow under field conditions is not yet possible in a satisfactory way, because of the difficulties involved in locating the roots and in measuring the boundary conditions at the root surface. The flow of water above the groundwater table is controlled by the physical properties of the soil. Knowledge of these properties is necessary in this field of hydrological studies. A practical method for the determination of the capillary conductivity is therefore needed.

GARDNER (1956) presents a method to calculate the capillary conductivity from pressure plate outflow data. He assumes that the slope of the moisture content - suction curve and the capillary conductivity for small increments of the suction may be taken as being constant. By measuring the outflow, the capillary conductivity can be calculated from the solution of the differential equation given by GARDNER.

Difficulties arise, however, if the impedance of the membrane or the sinter cannot be neglected. In this case too small values of the capillary conductivity will be calculated. Miller and Eurick (1958) give a method for the calculation of the capillary conductivity with non-negligible membrane impedance. As is pointed out by the authors, the principal limitation of their procedure is the uncertainty present in the determination of the flow impedance of the membrane. Unless good contact is established between the membrane and the soil, an unknown contact impedance may exist, which can have an important magnitude in comparison with the flow impedance of the

1) Received for publication March 21, 1959. 
membrane itself. Only the latter can be determined experimentally. Further, the impedance of the membrane or sinter can change during the experiments by plugging of the larger pores by dirt, bacterial growth and swelling or shrinking.

It is the purpose of this paper to give a method to calculate the capillary conductivity of the soil from pressure plate outflow data for non-negligible membrane impedances in which it is not necessary to determine the value of the membrane impedance experimentally. Therefore a method was developed to determine the sum of all impedances occurring at the lower boundary of the sample from the outflow data.

Theoretical analysis ${ }^{2}$ )

According to Richards (1931) the unsaturated flow can be described by DARCY's law:

$$
V=-K_{(\Theta)} \frac{d \Phi}{d z}
$$

where $V$ is the volumetric flow velocity, $\frac{d \Phi}{d z}$ is the potential gradient, $\Theta$ is the volume of water per unit volume of soil and $K_{\Theta}$ is the capillary conductivity. This conductivity is not a constant but a function of the moisture content of the soil and therefore also of the suction of the soil. Combining Darcy's law with the law of conservation of matter gives:

$$
\frac{\delta \Theta}{\delta t}=\frac{\delta}{\delta z}\left(K_{(\Theta)} \frac{\delta \Phi}{\delta z}\right)
$$

Consider a sample of soil having a volume $V$, a cross-sectional area $A$ and a height $l$, which is placed in a pressure membrane apparatus. Suppose the initial pressure in the chamber being $P^{i}$ and the moisture in the soil being in equilibrium with the atmosferic air-pressure outside the chamber. At time $t=0$ the pressure in the chamber is increased by a small amount $\triangle P$, the final pressure being: $P_{f}=P_{i}+\triangle P$. Due to the increment of the pressure water will flow out of the soil until equilibrium is attained again. The differential equatiton for the one-dimensional vertical flow under discussion is :

$$
\frac{\delta \Theta}{\delta t}=\frac{\delta}{\delta z} K_{\Theta}\left(\frac{\delta P}{\delta z}+1\right)
$$

In general this equation is non-linear and difficult to solve analytically. Following Gardner (1956) it is assumed that between $P_{i}$ and $P_{i}+\triangle P$ the capillary conductivity is a constant and that there exists a linear relationship between $P$ and $\Theta$ in this range of pressures, provided that the increments of $\triangle P$ are sufficiently small.

Now the relation between the soil moisture content and the pressure is given by:

$$
\Theta_{(P)}=a+b P
$$

where $a$ and $b$ are constants.

2) The author wishes to express his gratitude to dr. J. Wesselno for his helpful criticism. 
Neglecting gravity and substituting (4) into (3) gives:

$$
\frac{\delta P}{\delta t}=D \frac{\delta^{2} P}{\delta z^{2}}
$$

where

$$
D=K / b
$$

As no flow of water exists across the upper boundary of the sample, the potential gradient must vanish at this plane, thus :

$$
\frac{\delta P}{\delta z}=0 \quad z=0
$$

Assuming that the flow of water through the cross-sectional area at $z=l$ equals the flow through the membrane at each time, the lower boundary condition will be :

$$
K \frac{\delta P}{\delta z}+\frac{K_{p}}{l_{p}} P=0 \quad z=l
$$

where $P$ is the pressure drop through the membrane, $K_{p}$ is the hydraulic conductivity of the membrane and $l_{p}$ is the thickness of the membrane.

The initial condition requires:

$$
P=\triangle P \quad 0 \leqslant z \leqslant l \quad t=0
$$

Particular solutions of eq. (5) satisfying the condition (6) have the general form :

$$
P=B e^{-D \alpha^{2} t / l^{2}} \cos \alpha \frac{z}{l}
$$

where $\alpha$ is any dimensionless constant. Condition (7) is satisfied when :

in which $h=\frac{K_{p}}{l_{p} K}$

$$
\frac{K_{p}}{l_{p} K} l=h l=\alpha \tan \alpha
$$

In order to satisfy the condition (8) the coefficients $B_{n}$ of the infinite Fourier series, giving the general solution of eq. (5) must be determined. Multiplying through the series by $\cos \alpha_{n} \frac{z}{l}$ and integrating from 0 to $l$, yields :

$$
B_{n}=\frac{2 \Delta P \sin \alpha_{n}}{\cos \alpha_{n} \sin \alpha_{n}+\alpha_{n}}
$$

where $\alpha_{n}$ is the $n$th root of eq. (10). The first six roots are given by CARSLAW and JAFGER (1959) appendix IV, table I. With the aid of eq. (10) $B_{n}$ can be changed into :

$$
B_{n}=\frac{2 \triangle P h l \sec \alpha_{n}}{h l(\overline{h l}+1)+\alpha_{n}^{2}}
$$

Substituting this form into eq. (9) the general solution of eq. (5) subject to the conditions $(6),(7)$ and (8) becomes:

$$
P=2 \triangle P \sum_{n=1}^{\backsim} \frac{h l \sec \alpha_{n}}{h l(h l+1)+\alpha_{n}^{2}} e^{-D \alpha_{n}^{2} t / l^{2}} \cos \alpha_{n} \frac{z}{l}
$$


Substitution of (12) into (4) gives:

$$
\Theta_{(z t)}=a+2 b \triangle P \sum_{n=1}^{\backsim} \frac{h l \sec \alpha_{n}}{h l(h l+1)+\alpha_{n}{ }^{2}} e^{-D \alpha_{n}{ }^{2} t / l^{2}} \cos a_{n} \frac{z}{l}
$$

In order to find the total volume of water $\left(W_{t}\right)$ remaining in the soil sample at any time $t$, eq. (13) is multiplied through by the cross-sectional area $A$ and integrated from 0 to $l$, thus:

$$
W_{t}=\int_{o}^{l} \Theta_{(z t)} A d z=a V+2 b V \Delta P \sum_{n=1}^{\infty} \frac{h^{2} l^{2}}{\alpha_{n}^{2}\left[h l(h l+1)+\alpha_{n}^{2}\right]} e^{-D \alpha_{n}{ }^{2} t \mid l^{2}}
$$

The initial volume of water $\left(W_{i}\right)$ in the soil sample derived from eq. (14) at $t=0$ is :

$$
\mathrm{W}_{i}=a \mathrm{~V}+b \mathrm{~V} \triangle \boldsymbol{P}
$$

and the volume of water remaining in the soil at time $t=n$ is :

$$
\mathrm{W}_{f}=a \mathrm{~V}
$$

The total outflow, which can be extracted from the soil between $P_{i}$ and $P_{f}$ is :

$$
Q_{o}=W_{i}-W_{f}=b V \triangle P
$$

Solving for $b$ gives :

$$
b=\frac{Q_{o}}{V \triangle P}
$$

Equation (17) allows the calculation of $b$ from the total outflow $Q_{0}$, the volume of the sample $V$ and the pressure increment $\triangle P$.

The outflow at any time $t$ is : $\mathscr{Q}_{t}=W_{i}-W_{t}$. Combining this relation with (14), (15) and (16) yields :

$$
\frac{Q_{o}-Q_{t}}{Q_{0}}=2 \sum_{n=1}^{\infty} \frac{h^{2} l^{2}}{\alpha_{n}^{2}\left[h l(h l+1)+\alpha_{n}^{2}\right]^{-D \alpha^{2} t / l^{2}}}
$$

This infinite series converges rapidly and for $t>0.3 l^{2} / \alpha^{2} D$ the second term is less than $1 \%$ of the first. Neglecting all terms of the series except the first one and taking logarithms gives:

$$
{ }^{10} \log \frac{Q_{0}-Q_{t}}{Q_{0}}={ }^{10} \log \frac{2 h^{2} l^{2}}{\alpha_{1}{ }^{2}\left[h l(h l+1)+\alpha_{1}{ }^{2}\right]}-0.4343 D \alpha_{1}{ }^{2} t / l^{2}
$$

Plotting now $\log \frac{Q_{0}-Q_{t}}{Q_{0}}$ against $D t / l^{2}$ eq. (19) yields straight lines for various values of $h l$ (fig. 1). The dotted line in fig. 1 represents the relation given by GARDNER (1956) where $h l$ is infinite. The first part at the right hand side of eq. (19) gives the $\log \frac{Q_{0}-Q_{t}}{Q_{0}}$ intercepts, which are independent of the scale of $D t / l^{2}$. 


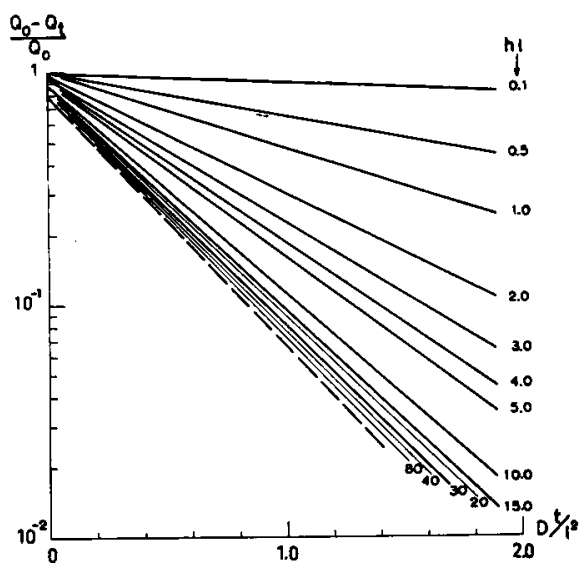

Fig. 1 The relation Between $\frac{Q_{o}-Q_{t}}{Q_{o}}$ and $D t / l^{2}$ for various values of $h l$. The dotted LiNe gives GardNER's solution, Without MEMBrane IMPEDance.

\section{Procedure}

The amount $\frac{Q_{o}-Q_{t}}{Q_{0}}$ is computed from the experimental values of the outflow $Q_{i}$ and from the final equilibrium-yield $Q_{0}$. The values of $\log \frac{Q_{0}-Q_{i}}{Q_{0}}$ are plotted against $t / l^{2}$ and a straight line is drawn through the experimental points. The slope of this line being

$$
\tan \beta^{\prime}=-0.4343 D \alpha_{1}^{2}
$$

is determined.

The slope of the lines in fig. 1 is given by:

$$
\tan \beta=-0.4343 \alpha_{1}^{2}
$$

Eq. (20) and (21) only differ by the unknown diffusivity $D$ of the soil. This simply means an enlargement or reduction of the $D t / l^{2}$-scale, dependent on the value of $D$. In order to find $D$ we consider the interception point of the straight line with the $\frac{Q_{0}-Q_{t}}{Q_{0}}$-axis. This point is not influenced by changing the scale of $D t / l^{2}$. The value of the $\log \frac{Q_{0}-Q_{t}}{Q_{0}}$ intercept is given by the first part at the right hand side of eq. (19) and is only dependent on the value of $h l$ as can be seen from eq. (10). As the intercept, defined in this way, is the same for any $D t / l^{2}$-scale, it can be determined from the straight line connecting the experimental points. With the known intercept, the corresponding value of $h l$ can be determined. For this purpose the relation between the intercept and $h l$ is plotted in fig. 2. With the aid of this figure the value of $h l$ can easily be read for each value of the intercept. A determination of the membrane impedance is therefore unnecessary.

The next step is finding the value of $\tan \beta$ corresponding with the $h l$-value. In order to simplify this step, the relation between the value of $h l$ and $\tan \beta$. derived from eq. (10) and (19), is plotted in fig. 3. 


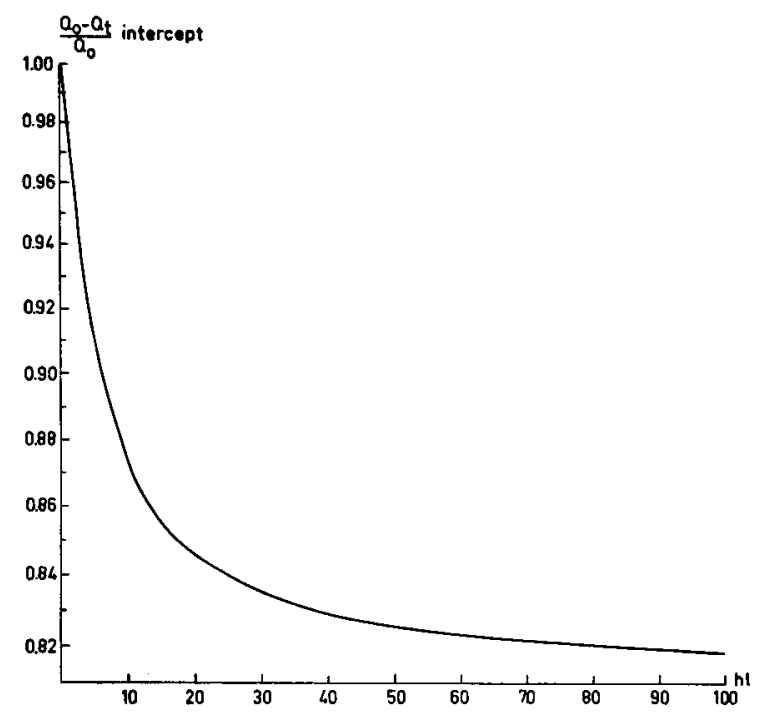

Fig. 2 The relation Between the intercept of the $\frac{Q_{o}-Q_{t}}{Q_{0}}$-axis and $h l$.

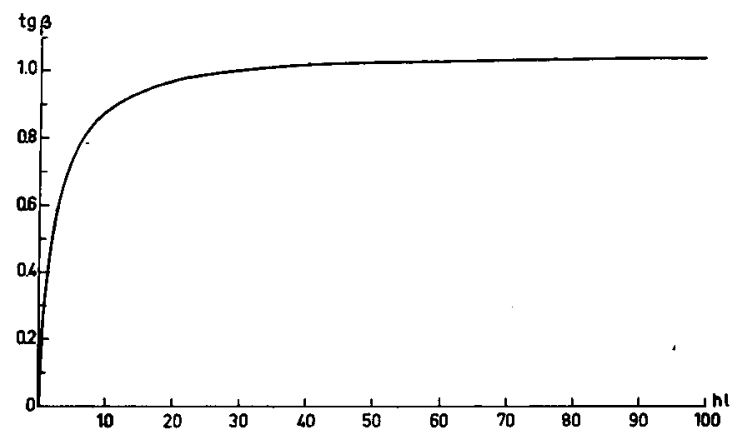

Fig. 3 The relation between the SLOPE OF the LINES Given iN Fig. 1 and $h l$.

The value of $\tan \beta$, corresponding with the $h l$-value, can now be read from this figure. Dividing $\tan \beta^{\prime}$ by the value of $\tan \beta$ yields the desired value $D$ of the soil. The capillary conductivity can now be computed by combining eq. (5a) and (17) which gives :

$$
K=\frac{D Q_{0}}{V \triangle P}
$$

\section{Discussion}

The possibility of refining GardNER's solution is fully dependent on the accuracy of the outflow data since these control the exactness with which the $\log \frac{Q_{0}-Q_{t}}{Q_{0}}$-intercepts can be determined. The accuracy of the intercepts, controls - in its turn - the exact value of $h l$. The small differences between the intercepts for various values of $h l$ do not imply that the correction for the membrane impedance is correspondingly small. When the membrane impe- 
dance is neglected the calculated values of the capillary conductivity generally will be too small. With the aid of eq. (19) the influence of the membrane impedance can be calculated. In table 1 the capillary conductivity, calculated with neglected membrane impedance is expressed in per cents of the real capillary conductivity, which will be found when the membrane impedance is taken into account.

Table I The calculated capillary conductivity by neglecting the membrane impedance in per cents of the real value for various values of $h l$.

\begin{tabular}{l|r|r|r|r|r|r|r}
\hline$h l$ & $\%$ & $h l$ & $\%$ & $h l$ & $\%$ & $h l$ & $\%$ \\
\hline 0.1 & 4.2 & 2.0 & 47.2 & 5 & 69.9 & 30 & 94.0 \\
0.5 & 17.3 & 3.0 & 57.7 & 10 & 83.0 & 50 & 96.3 \\
1.0 & 30.4 & 4.0 & 65.0 & 20 & 91.0 & 100 & 98.2 \\
\hline
\end{tabular}

For $h l=20$ the capillary conductivity obtained by neglecting the membrane impedance is $9 \%$ too small. If one wishes to determine the capillary conductivity with a certain accuracy, neglecting the membrane impedance, the desired hydraulic conductivity of the membrane can be derived from table 1 and eq. (10). The contact impedance between the soil sample and the membrane, however, remains unknown and therefore such a test is of limited practical value.

In comparison with the method given by MILLER and ELrick (1958), the method proposed in this paper has the advantage that it is unnecessary to determine the membrane impedance experimentally. This is due to the fact that the $h l$-value determined from the $\frac{Q_{o}-Q_{t}}{Q_{o}}$ intercept is an indirect value for the flow impedance occurring during the experiment. Any contact impedance, if present, between the soil sample and the membrane automatically implies a change in the $h l$-value determined. Changes in the membrane impedance due to plugging of the pores, bacterial growth and swelling or shrinking are accounted for, as the $h l$-value is determined anew for each pressure step applied. Errors due to inaccurate matching of scales are avoided.

\section{LITERATURE}

CARsLaW, H. S. and J. C. JAEGER : Conduction of heat in solids. Oxford at the Clarendon Press (1959).

GARDNER, W. R.: Calculation of capillary conductivity from pressure plate outflow data. Soil Sci. Soc. Am. Proc. 20 (1956) 317-320.

Miller, E. E. and D. E. Elrick : Dynamic determination of capillary conductivity extended from non-negligible membrane impedance. Soil Sci. Soc. Am. Proc. 22 (1958) 483-486.

Rrchards, L. A. : Capillary conduction of liquids through porous media. Physics 1 (1931) $318-333$. 